\title{
You don't need a mentor; you need a board of directors
}

\author{
Jonathan Sherbino, MD MEd*
}

The history of medical education is built on apprenticeship. From Hippocrates's followers to the medieval guilds to Harvey Cushing and William Osler ${ }^{1}$, the concept of a novice gaining experience under the guidance of an expert is embedded in medicine. With many points of overlap with apprenticeship, receiving and providing mentorship is central to the profession of medicine. Mentorship is a professional relationship, where a more experienced individual shares wisdom (e.g. tacit knowledge that has been acquired through experience), guides, advocates, and champions a less experienced individual. In one review, between $19-93 \%$ of academic physicians reported being mentored. ${ }^{2}$ In this issue of $C F E M$, Fernando et al. describe the results of an on-line survey of Canadian emergency physicians (EPs) experience with mentorship during residency training. ${ }^{3}$

Developing as an emergency physician requires significant guidance. The objectives of mentorship programs in medicine include to: improve clinical practice, guide career development, promote retention, increase collaboration, promote organizational culture, develop professional networks, attract trainees to a specialty, increase success in academic promotion, provide specific project guidance, improve research success in grant acquisition and publication, improve academic writing, improve teaching practice, develop curricula, provide emotional support, foster gender equity and foster racial equity. ${ }^{2,4,5}$ Clearly, the goals of a mentoring relationship can be quite ambitious, but more likely, are tailored to the needs of a mentee. Of course, this presupposes that a mentee can clearly articulate and define their professional needs and appreciate the interconnected nature of some of the objectives described above.

The classic archetype of mentorship is the dyad Telemachus and Mentor (see Homer's Odyssey, I'm very sorry ${ }^{6}$ ) - the novice and the expert. Among the many interesting findings from Fernando et al., hidden in Table 3 is data that indicates two-thirds of respondents had more than one mentor. This is an important and telling result; one that needs more. While the literature includes multiple mentoring models, including speed mentoring, group mentoring, and peer mentoring, among others, the most common, and often exclusive model is the dyad. Too frequently the assumption for mentees, mentors, and mentorship programs is that the complex nature of mentorship is best achieved through the full service, classic, dyad relationship. But, you don't need a mentor; you need a board of directors.

\section{THE COMPLEX ADVICE AND ADVOCACY REOUIRED TO DEVELOP AS AN EMERGENCY PHYSICIAN CAN RARELY BE PROVIDED BY A SINGLE INDIVIDUAL.}

When you consider the many elements that inform physician practice, the expectation that a single individual will possess the necessary wisdom in all of these domains is unrealistic. Guidance regarding EM practice, subspecialty clinical practice, leadership, administration, academic contributions, scholarship, wellness, and more is complex and distinct within each domain. It is the rare physician that has mastered all of these areas. The savvy mentee will appreciate that mentors can be drawn from outside of emergency medicine, or even medicine, if the wisdom sought does not require a medical context. Moreover, for each individual, and especially for underrepresented, marginalized and disadvantage groups, the contextual nuance of advice requires an individual who understands and has navigated discriminatory barriers in the specific clinical or academic environment. In a manner similar to the numerous consultations required for a complex patient or the board

From the *Professor of Emergency Medicine, Assistant Dean of Health Professions Education Research, McMaster University.

Correspondence to: David Braley Health Sciences Centre, Room 5003, 1280 Main St W. Hamilton, ON L8S 4K1; Email: sherbino@mcmaster.ca; Twitter: @sherbino 
of a directors that advises the chief executive officer of a company, a robust and effective mentoring relationship is rarely (if ever) a dyad. Rather, an interconnected network of several mentors, each with unique and specialized perspectives, provides the mentee with more robust, effective and contextually-relevant counsel.

In addition, diffusing the responsibilities of mentorship across multiple individuals, allows mentors to more readily commit to a mentoring relationship. Rather than solo, heavy lifting (e.g. full service mentoring), the responsibility for sustained investment in a mentee is shared among the board of directors. For heavily committed and highly desirable mentors, the reduced time commitment may be more feasible. ${ }^{7}$ For the mentee, multiple mentors ensure more timely and regular access. No longer constrained by a single individual's schedule, multiple mentors may improve responsiveness, provided that there is some cross over in counsel between the domains or role that each mentor theoretically represents.

\section{TECHNOLOGY TRUMPS GEOGRAPHY; DO NOT LIMIT THE POOL OF POTENTIAL MENTORS TO LOCAL PHYSICIANS, LEADERS OR ACADEMICS.}

Of course, if finding a single mentor is hard, finding multiple mentors can be truly problematic, if the pool is constrained by individuals associated with local institutions. With the rapid development of free, high quality, stable videoconferencing and other on-line communication platforms the potential mentorship pool is exponentially expanded. Current digital and online communication platforms are able to closely approximate in-person meetings.

Accessing this pool of potential digital mentors still requires the challenging negotiation of establishing a relationship. Neither directly approaching a potential mentor as a mentee, nor using an intermediary, is obviously preferential. ${ }^{8,9}$ Yet, a digital mentor is not necessarily an unknown, connected either via a personal virtual professional network or the professional network of an existing member of the mentee's board of directors. The rise of virtual communities of practice via online discussion groups, Twitter chats, and free openaccess medical education (\#FOAMed) forums has allowed legitimate peripheral participation of early career physicians to engage with established core members (i.e., experts). ${ }^{10}$ Connecting with a potential mentor through initial participation in a virtual community of practice is occurring with increasing frequency. One caveat of course is that the inclusion of digital mentors in a board of directors still requires at least one individual familiar with the context and politics of the local institution of the mentee.

Finally, where does this preferred representation of mentorship via a board of directors, in contrast to a dyad model, lead? Rather than arithmetic, linear growth of a mentee's professional network, a board of directors leads to exponential, interconnected growth of a much larger professional network. With a board of directors, including digital mentors, a professional network quickly grows, and the global community of emergency medicine begins to shrink.

Keywords: continuing professional development, digital, education, mentor

Acknowledgements: Many thanks to Dr. Teresa Chan for advice on the development of this commentary.

\section{REFERENCES}

1. Duffy TP The Osler-Cushing covenant. Perspect Biol Med 2005;48(4):592-602.

2. Sambunjak D, Straus SE, Marusić A. Mentoring in academic medicine: a systematic review. FAMA 2006;296(9):1103-5

3. Fernando SM, Cheung WJ, Choi SB, Thurgur L, Frank JR. Faculty mentorship during residency and professional development among practising emergency physicians. CFEM 2018

4. Sambunjak D, Straus SE, Marusic A. A systematic review of qualitative research on the meaning and characteristics of mentoring in academic medicine. 7 Gen Intern Med. 2010;25 (1):72-8.

5. Kashiwagi DT, Varkey P, Cook DA. Mentoring programs for physicians in academic medicine: a systematic review. Acad Med 2013;88(7):1029-37.

6. https://en.wikipedia.org/wiki/Mentor_(Odyssey) Accessed September 27, 2018.

7. Shen Y, Cotton RD, Kram KE. Assembling your personal board of advisors. MIT Sloan Manag Rev 2015;2:81-90.

8. Coates WC. Being a mentor: what's in it for me? Acad Emerg Med 2012;19(1):92-7.

9. Ramani S, Gruppen L, Kachur EK. Twelve tips for developing effective mentors. Med Teach 2006;28(5):404-8.

10. Gottlieb M, Fant A, King A, Messman A, Robinson D, Carmelli G, Sherbino J. One Click Away: Digital Mentorship in the Modern Era. Cureus 2017;9(11):e1838. 